\title{
SUPERTWIN: towards 100kpixel CMOS quantum image sensors for quantum optics applications
}

Leonardo Gasparini, Bänz Bessire, Manuel Unternährer, André Stefanov, Dmitri Boiko, et al.

Leonardo Gasparini, Bänz Bessire, Manuel Unternährer, André Stefanov, Dmitri Boiko, Matteo Perenzoni, David Stoppa, "SUPERTWIN: towards 100kpixel CMOS quantum image sensors for quantum optics applications," Proc. SPIE 10111, Quantum Sensing and Nano Electronics and Photonics XIV, 101112L (27 January 2017); doi: 10.1117/12.2253598

SPIE. Event: SPIE OPTO, 2017, San Francisco, California, United States 


\title{
SUPERTWIN: towards 100kpixel CMOS quantum image sensors for quantum optics applications
}

\author{
Leonardo Gasparini ${ }^{\mathrm{a}}$, Bänz Bessire ${ }^{\mathrm{b}}$, Manuel Unternährer ${ }^{\mathrm{b}}$, \\ André Stefanov $^{\mathrm{b}}$, Dmitri Boiko ${ }^{\mathrm{c}}$, Matteo Perenzoni ${ }^{\mathrm{a}}$, David Stoppa ${ }^{\mathrm{a}}$ \\ ${ }^{a}$ Fondazione Bruno Kessler, via Sommarive 18, Povo, 38123 Trento, Italy; ${ }^{b}$ Institute of Applied \\ Physics, University of Bern, 3012 Bern, Switzerland; ${ }^{\mathrm{c}}$ Centre Suisse d'Électronique et de \\ Microtechnique, Jaquet-Droz 1, CH-2002 Neuchâtel, Switzerland
}

\begin{abstract}
Quantum imaging uses entangled photons to overcome the limits of a classical-light apparatus in terms of image quality, beating the standard shot-noise limit, and exceeding the Abbe diffraction limit for resolution. In today experiments, the spatial properties of entangled photons are recorded by means of complex and slow setups that include either the motorized scanning of single-pixel single-photon detectors, such as Photo-Multiplier Tubes (PMT) or Silicon PhotoMultipliers (SiPM), or the use of low frame rate intensified CCD cameras. CMOS arrays of Single Photon Avalanche Diodes (SPAD) represent a relatively recent technology that may lead to simpler setups and faster acquisition. They are spatially- and time-resolved single-photon detectors, i.e. they can provide the position within the array and the time of arrival of every detected photon with $<100$ ps resolution. SUPERTWIN is a European H2020 project aiming at developing the technological building blocks (emitter, detector and system) for a new, all solid-state quantum microscope system exploiting entangled photons to overcome the Rayleigh limit, targeting a resolution of 40nm. This work provides the measurement results of the $2^{\text {nd }}$ order cross-correlation function relative to a flux of entangled photon pairs acquired with a fully digital $8 \times 16$ pixel SPAD array in CMOS technology. The limitations for application in quantum optics of the employed architecture and of other solutions in the literature will be analyzed, with emphasis on crosstalk. Then, the specifications for a dedicated detector will be given, paving the way for future implementations of 100kpixel Quantum Image Sensors.
\end{abstract}

Keywords: Single-photon detector, quantum imaging, entangled photons, single-photon avalanche diode, crosstalk, entanglement-enhanced microscope, super-resolution, all-solid-state microscope

\section{INTRODUCTION}

Arrays of SPADs implemented in standard CMOS technology are a relatively recent development in single-photon detection. With respect to other technologies such Photo-Multiplier Tubes (PMT) and Silicon Photo-Multipliers (SiPM), CMOS SPAD arrays provide spatial resolving capabilities at high resolution in addition to sub-nanosecond temporally resolving capabilities. For this reason, they represent a valid alternative to Electron-Multiplying CCDs (EMCCD).

So far, CMOS SPAD technology has been applied (i) in biology for Fluorescence Lifetime Imaging Microscopy (FLIM) [1] and Raman Spectroscopy [2]; (ii) in the medical field for Positron Emission Tomography (PET) [3]; (iii) in the industrial/automotive/space domains for 3D Time-of-Flight (3D ToF) measurements $[4,5,6]$; and (iv) in cryptography for Quantum Random Number Generation (QRNG) [7]. Recently, CMOS SPAD consumer products entered the market as Light Detection And Ranging (LiDAR) devices for smartphone camera autofocusing [8].

The technology has evolved through the years, and it is now mature for being adopted in quantum physics experiments to optimize setups and enable new types of investigation. In particular, quantum physics experiments require high spatial resolution, high sensitivity in the visible range up to the 
Near Infra-Red (NIR) region, sub-100ps time resolution, low dark count rates (DCR), negligible crosstalk and usability.

SUPERTWIN [9] is a project funded by the European Commission within the Horizon 2020 programme that involves the development of a new concept of quantum microscope employing all solid-state technologies for the generation and recording of entangled photons. The design of SPAD arrays in CMOS technology customized to capture spatio-temporal multi-photon correlation functions is one of the project main goals. This work describes the use of a CMOS SPAD array for measurement of the spatial second-order correlation function of entangled two-photon states in the far field. The sensor that has been used in this experiment is the SPADnet-I sensor, an $8 \times 16$ pixel SPAD-based CMOS imager [3]. The experiment shows the technology strengths and weaknesses. A roadmap towards the development of high-resolution CMOS quantum image sensors for quantum optics applications is then provided.

The remainder of the paper is organized as follows. We provide an overview on state-of-the-art of SPAD arrays implemented in CMOS technology in Sec. 2. We describe the experiment in Sec. 3, summarizing the architecture and performance of the SPADnet-I sensor, along with the description of the optical setup and the results of the experiment. The effect of crosstalk in the SPADnet-I sensor is analyzed in Sec. 4. Finally, we provide a projection on the next generation of CMOS SPAD arrays for quantum optics imagers in Sec. 5.

\section{STATE OF THE ART IN CMOS SPAD ARRAYS}

The state-of-the-art in terms of spatial resolution for a SPAD-based sensor is represented by [10] that reports a QVGA 8- $\mu \mathrm{m}$ pixel pitch with $26.8 \%$ fill factor (FF). However its time-resolving performance is in the order of nanoseconds and not fully reported yet while the sensor frame rate is of $16 \mathrm{kfps}$.

[1] reports a time-gated $512 \times 128$-pixel imager fabricated in standard high-voltage $0.35 \mu \mathrm{m}$ CMOS technology. The pixels of $24 \mu \mathrm{m}$ pitch include a SPAD and its front-end and the electronics required for time gating and a 1-bit memory to store the acquired data (photon detected or not detected). The sensor achieves a pixel FF of 5\% and a maximum frame rate of $156 \mathrm{kfps}$. The minimum gating width is $4 \mathrm{~ns}$. The sensor has been applied to fluorescence lifetime imaging applications and as a true random number generator.

In [11], authors characterize a linear 256×2-pixel sensor fabricated in standard 130nm CMOS process in a testbed for application in time-resolved fluorescence spectroscopy. Each pixel contains four time-gated SPADs and a Time-to-Digital Converter (TDC) that records the SPAD triggering time with 40ps resolution. The electronics fits in an area of $23.78 \times 100 \mu \mathrm{m}^{2}$, achieving a $43.7 \% \mathrm{FF}$. In addition, the chip includes the circuitry to perform per-pixel Time-Correlated Single-Photon Counting (TCSPC) with 320ps binning and center of mass calculation. The line rate of the sensor in TCSPC mode is $200 \mathrm{~Hz}$. The sensor total jitter is 700ps FWHM.

Direct Time-of-Flight (ToF) 3D imaging is another popular application of CMOS SPAD arrays. In [4], a fully digital $32 \times 32$-pixel imager fabricated in $0.35 \mu \mathrm{m}$ automotive certified process is presented. Each $150 \times 150 \mu \mathrm{m}^{2}, 3.14 \% \mathrm{FF}$ pixel can operate in photon-counting mode or photon-

timestamping mode, employing 310ps TDCs. A state-of-the-art DCR density of $0.17 \mathrm{~Hz} / \mu \mathrm{m}^{2}$ is 
reported, too. Frames are acquired at up to $100 \mathrm{kfps}$. The sensor timing performance in terms of jitter is of $\sim 600$ ps FWHM.

Another ToF sensor in high-voltage $0.35 \mu \mathrm{m}$ standard CMOS technology has been presented in [5]. It consists of a $9 \times 9$ matrix of SPADs having a $>40 \%$ FF. A $3 \times 3$ subset of SPADs is enabled at a time and coupled to an array of high-performance $(<10 \mathrm{ps}$ binning) TDCs, which can be read out at ate of few MHz. The timing resolution is of 170ps FWHM.

A $320 \times 240$ array of SPAD-based is reported in [10] for time-gated photon counting applications. The pixel pitch is of $8 \mu \mathrm{m}$ and achieves a $26.8 \%$ FF. Binary images are acquired at a rate of $16 \mathrm{kfps}$, while timing performance is not reported.

These SPAD-based sensors are good potential candidates for coincidence detection of spatially correlated photon pairs, however, they suffer from very low fill factors $[1,4]$, time-coincidence detection capability longer than $0.6 \mathrm{~ns}[1,11,4]$, acquisition frame rates below $160 \mathrm{kHz}[1,11,4,5]$, limited spatial resolution of the $2 \mathrm{D}$ pixel array arrangement $[1,11]$.

The trend in CMOS SPAD-array design goes towards:

(i) the miniaturization of the pixel (below $10 \mu \mathrm{m}$ ) to increase the output image resolution;

(ii) SPAD optimization to improve the photon detection efficiency (PDE) while reducing DCR, after-pulsing and crosstalk;

(iii) 3D stacking of chips, with a top tier optimized for sensing that includes the array of SPADs and a bottom tier optimized for processing (i.e., counting, timestamping and buffering);

(iv) smart mechanisms for timestamping photons, such TDC sharing and time-gated counting in the analog domain;

(v) the on-chip implementation of pre-processing stages, such as timestamp histogramming, to reduce the sensor output data size and increase the frame rate, thus enabling synchronization with fast sources of photons (from $100 \mathrm{kHz}$ up to tens of $\mathrm{MHz}$ ).

\section{EXPERIMENT}

We conducted a preliminary study consisting in the acquisition with an existing SPAD-based imager of the second-order correlation patterns in the far-field of a flux of entangled photons.

SPADnet-I sensor is the sensor of choice for the experiment. It is a fully digital single-photon imager implemented in a 130nm CMOS process. It comprises an array of $8 \times 16$ pixels, and is fully synchronous with a global clock that defines the sampling frequency. Each pixel of $610.5 \times 571.2 \mu \mathrm{m}^{2}$ area includes an array of $24 \times 30$ SPADs whose outputs are digitized and merged into a single train of pulses which is fed to the pixel back-end logic. The back-end logic consists of digital counters, that count the number of photons within a clock bin, and TDCs, that record the time arrival of the first photon detected within a clock bin. The sensor can operate at up to $100 \mathrm{MHz}$. A micrograph of the chip is shown in Figure 1(a).

The sensor has been specifically designed for Positron Emission Tomography applications, and implements a number of additional features tailored for those purposes. Nevertheless, it includes several unique characteristics that make it suitable for quantum optics experiments, including (i) high pixel FF (42.6\%); (ii) per-pixel photon timestamping capabilities with 65ps resolution, 265ps jitter; (iii) high frame rate (up to 750kfps); and (iv) the capability to turn on/off SPADs individually, e.g., to calibrate the sensor and increase SNR by enabling only low noise devices. Figure 1(b) shows the acquisition timing applied in the quantum physics experiment. In this context, each SPADnet-I 
pixel captures one photon timestamp for two consecutive 10ns time bins before transmitting data to the external controller. The sensor readout takes less than $4 \mu$ s for the whole chip.

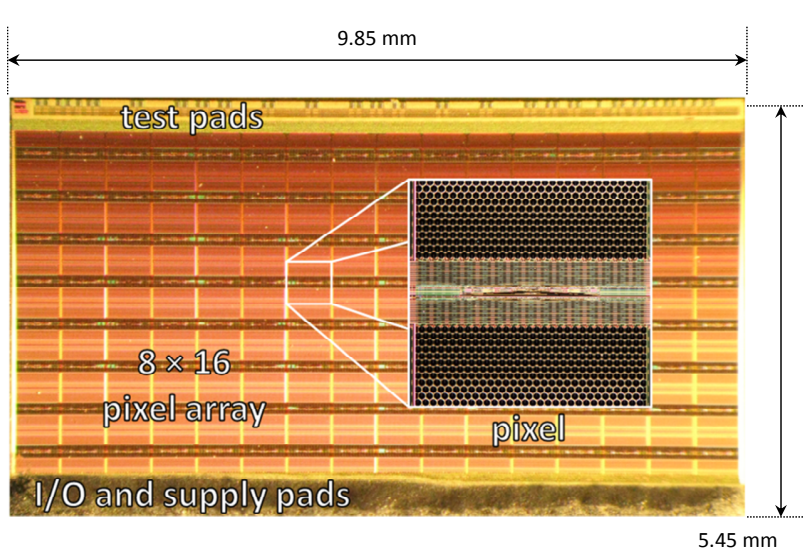

(a)

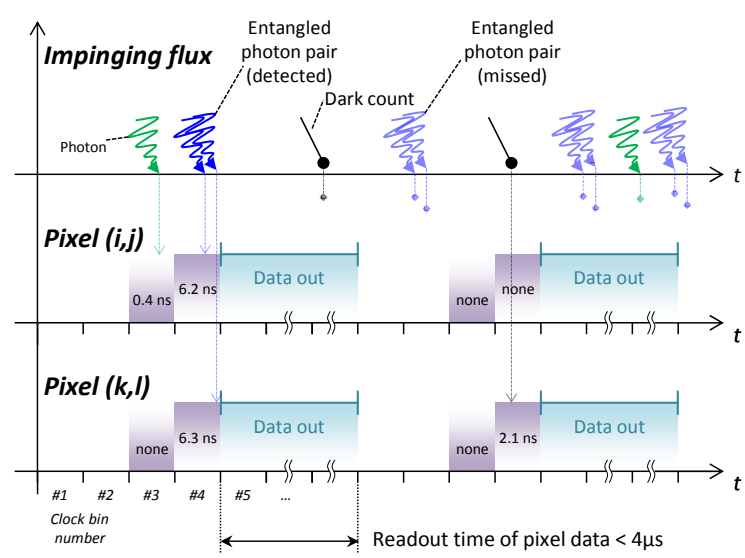

(b)

Figure 1 - (a) SPADnet-I chip micrograph. The inset shows a close-up on a single pixel, including 720 SPADs and, in the middle, the electronics required for calibration, SPAD operation, photon counting and photon timestamping. (b) SPADnet-I timing. In this context, the chip allows the user to record a timestamp per pixel in two consecutive clock bins. Then data are read out in less than $4 \mu \mathrm{s}$, with clock running at $100 \mathrm{MHz}$.

In the experiment, a non-linear crystal pumped with a continuous wave laser generates transverse momentum entangled photon pairs by means of Spontaneous Parametric Down-Conversion (SPDC). Under certain conditions, the photons are expected to exit the crystal with an angle with respect to the longitudinal axis, as shown in Figure 2. The experiment aims to record the spatial second-order correlation function in the far field.

Figure 3 shows the principle of two optical setups. Figure 3(a) describes the standard PMT- or SiPM-based approach, in which the spatial second-order correlation function is obtained by means of a 2D scan of the photon beam using two single-channel photodetectors; in Figure 3(b) the same measurement is performed by means of a CMOS SPAD-based imager.

The setup employs a $33 \mathrm{~mW}, 405 \mathrm{~nm}$ laser to pump the non-linear crystal. The temperature of the latter is chosen such that the entangled photons exit the crystal in a non-collinear configuration (see Figure 2). A set of filters is then used to remove the residual of the pumping laser and let only the pairs of entangled photons at $810 \mathrm{~nm} \pm 5 \mathrm{~nm}$ pass. A 50:50 beam splitter separates the beam into two beams. Typically, the two beams are coupled to optical fibers and provided as inputs to two singlechannel single-photon detectors, as in Figure 3(a). A 2D scan for the each of the channel is then required to fully characterize the photon flux, resulting in an $\mathrm{N}^{4}$ number of acquisition steps, where $\mathrm{N}^{2}$ is the resolution of the output image. In Figure 3(b) the scanning mechanism and the singlephoton detectors have been replaced by the SPADnet-I CMOS SPAD-based imager, capable of recording the arrival time of the detected photons individually for each pixel/channel. To improve signal-to-noise ratio, 50\% of the highest DCR SPADs have been disabled. On top of this, a ring of two-rows and two columns of dead SPADs have been created at the boundary of each pixel to reduce crosstalk at the expense of lowering the FF. 


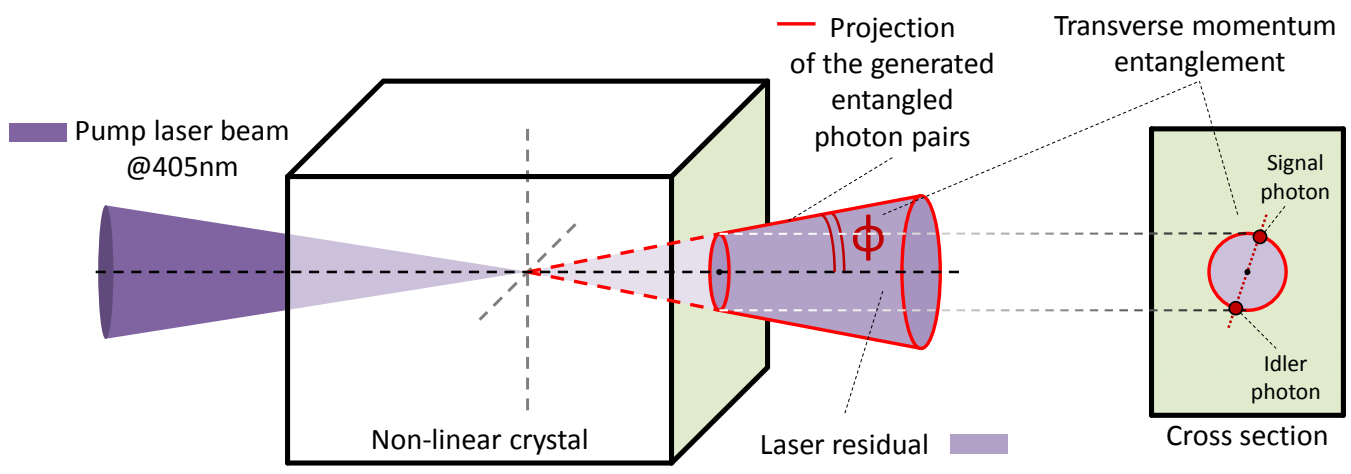

Figure 2 - Principle scheme of the interaction of the laser beam with the non-linear crystal. Transverse momentum entanglement is achieved by means of SPDC. In a classical configuration, the photon flux is split into two beams.

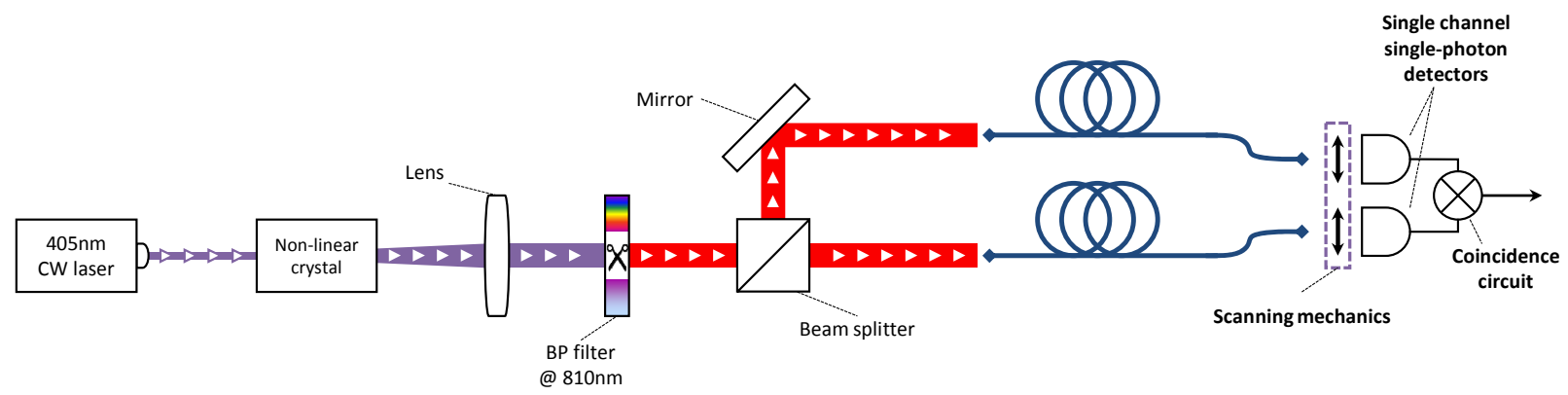

(a)
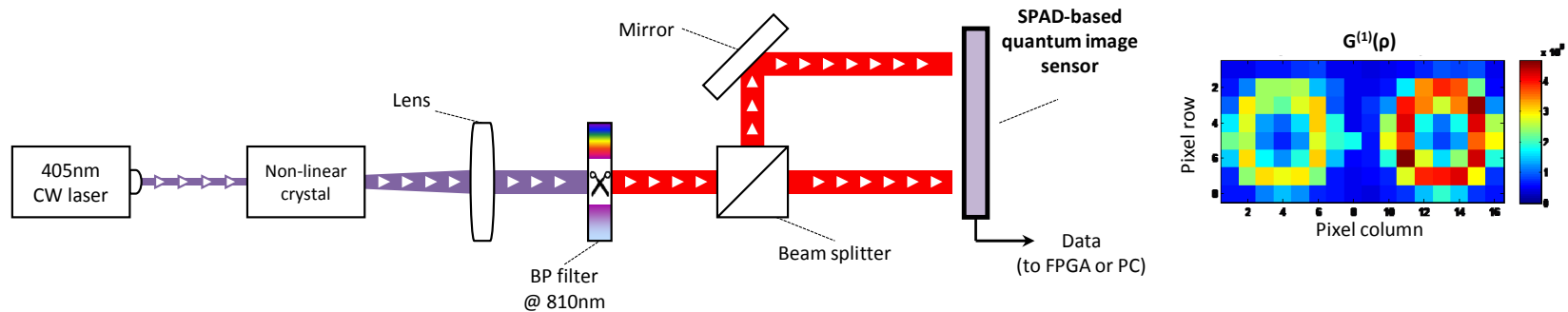

(b)

(c)

Figure 3 - Block diagram of the optical setup for spatial second-order correlation measurements of entangled (SPDC) twophoton states in far-field using (a) PMTs or SiPMs and (b) a CMOS SPAD array to record the photon statistics. The imaging plane lies in the focal plane of the lens. (c) represents the photon distribution on the sensor focal plane after several acquisitions, corresponding to the first-order correlation function. 


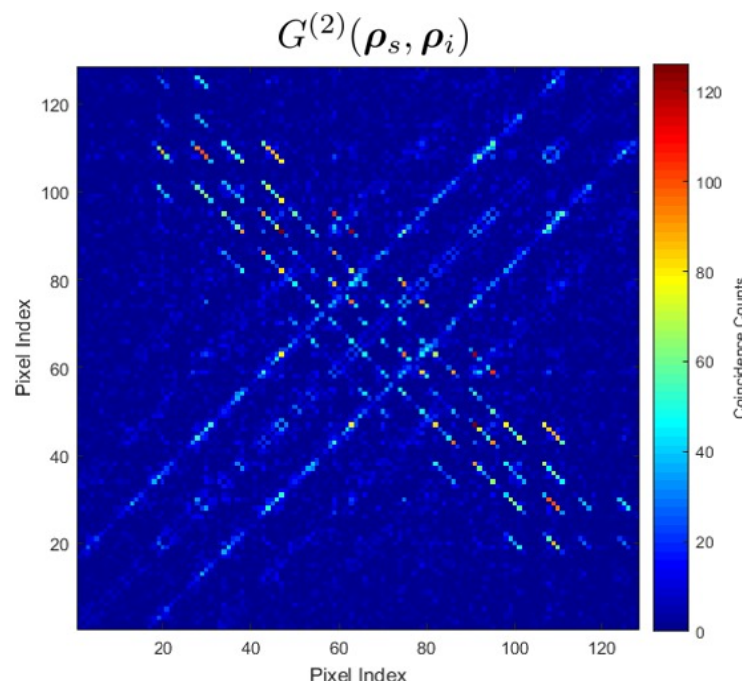

(a)

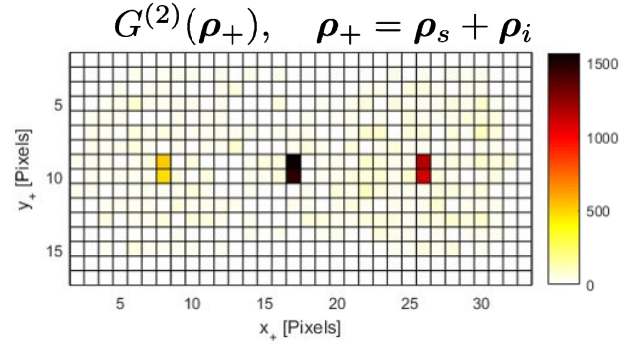

(b)

Figure 4 - Second-order correlation functions achieved with the given setup after $3 \mathrm{M}$ acquisitions. In $G^{(2)}\left(\rho_{s}, \rho_{i}\right)$, the NW-SE diagonals represents anti-correlation events, while NE-SW diagonals are correlation events caused by the crosstalk between neighboring pixels. $G^{(2)}\left(\rho_{+}\right)$shows three anticorrelation points, corresponding to the three possible outcomes of the beam splitting stage.

The experimental outcome is shown in Figure 3(c) and Figure 4. Figure 3(c) shows the first-order correlation function $G^{(1)}(\rho)$, extracted from the SPADnet-I sensor measurements simply accumulating three million acquisitions of $10 \mathrm{~ns}$ each. The uniform background is due to dark counts. The projection of the two split beams on the sensor focal plane is clearly visible on top of the uniform background noise.

In Figure 4(a), the photon arrival times recorded by the sensor have been processed to extract coincidence events and calculate the second-order correlation function $G^{(2)}\left(\rho_{s}, \rho_{i}\right)$. The sensor 2D coordinates have been linearized to display on each of the plot axis all the possible $128^{2}$ correlation points in a single graph. Pixel anti-correlations are visible along the NW-SE diagonal. On the opposite diagonal, lines represent false correlations attributed to crosstalk events, mostly visible at a distance of 16 pixels (i.e., one row).

Figure 4(b) shows the second-order correlation function $G^{(2)}\left(\rho_{+}\right)$, defined as:

$$
G^{(2)}\left(\rho_{+}\right)=\sum_{\rho_{s}+\rho_{i}=\rho_{+}} G^{(2)}\left(\rho_{s}, \rho_{i}\right)
$$

The plot highlights three anti-correlation points corresponding to each of the three possible outcomes of the beam splitting stage, namely (i) both the photons being transmitted (right), (ii) both being reflected (left), and (iii) one transmitted, one reflected (central anti-correlation point). It is worth mentioning that using the setup depicted in Figure 3(a), only the central anti-correlation point would be observed. Moreover, as the SPAD sensor generated a 2D pixel map of photon arrival times, we could also remove the beam splitter and directly focus the beam onto the sensor focal plane.

As in this experiment crosstalk-induced correlations behave differently with respect to the desired anticorrelations, they can be easily filtered out to produce a better anti-correlation plot. This is not true in general, though. For example, in a similar experiment in the near field, crosstalk events would not be distinguishable from the actual signal, and they would appear as artifacts on the processed image. 


\section{CROSSTALK ANALYSIS IN THE SPADNET-I SENSOR}

In order to have a better picture of the crosstalk problem in SPADnet-I, a large number of frames have been acquired in complete darkness. In this condition, all recorded events represent dark counts or crosstalk events. A histogram of time differences between any possible combinations of events recorded in the same frame has been generated. As dark counts are uncorrelated events, we do expect a uniform distribution of timestamps within a clock bin for every pixel. The histogram of time differences should then exhibit a triangular shape peaked at 0 , given by the convolution of two uniform distributions. Figure 5 shows the actual histogram obtained after many acquisitions. On top of the expected triangular shape, a peak at small time differences is observed. Clearly, this behavior is ascribed to crosstalk and can be analyzed by looking at spatial correlations.

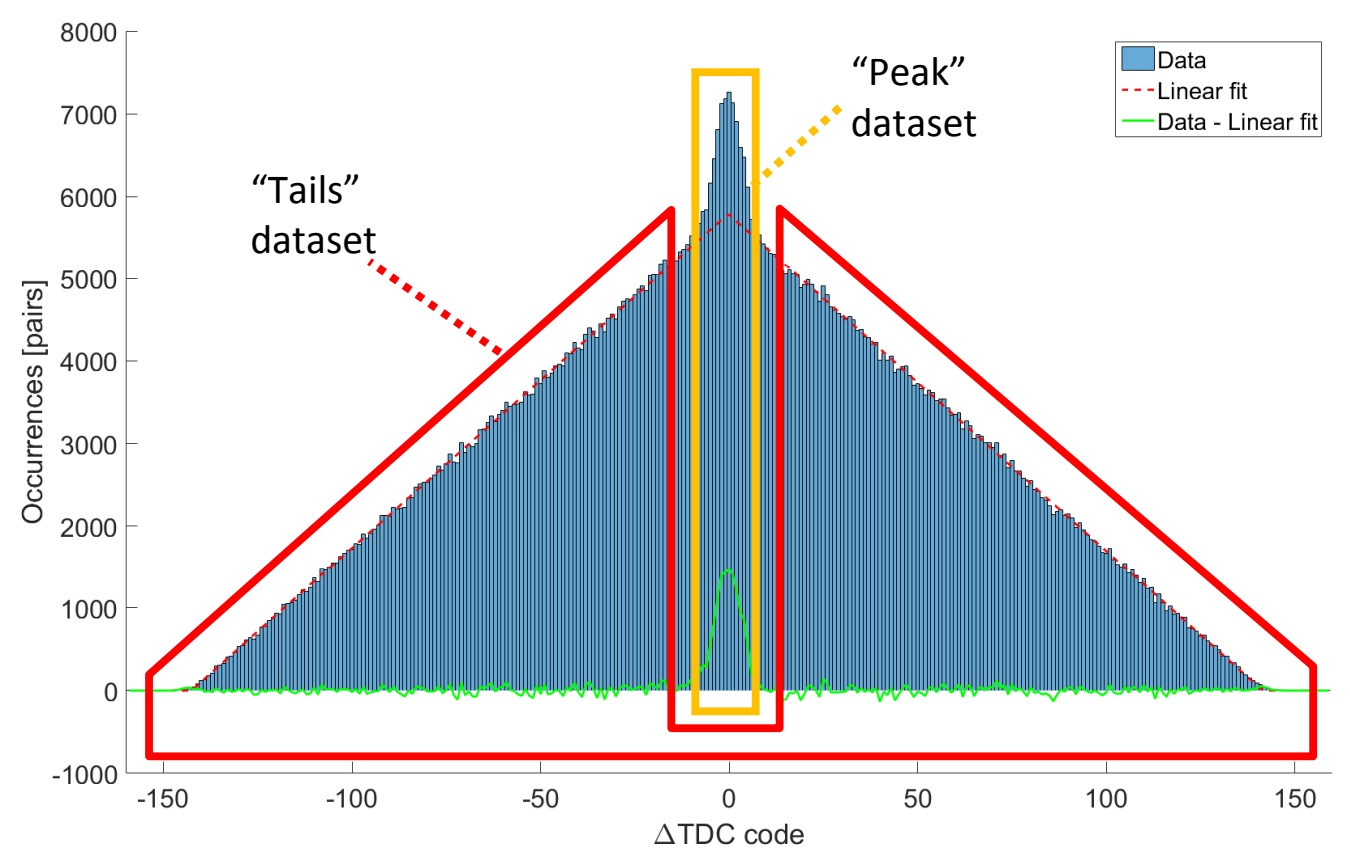

Figure 5 - Histogram of timestamp differences between noise events. On top of the expected triangular shape, an additive peak can be observed at values close to zero due to crosstalk. The green line represents the residual of data after removing the linear fit, and it corresponds to crosstalk induced events. The full dataset has been divided into the "tails" dataset, containing only dark count -generated timestamps, and the "peak" dataset, caused by accidentally simultaneous dark counts and crosstalk events. 


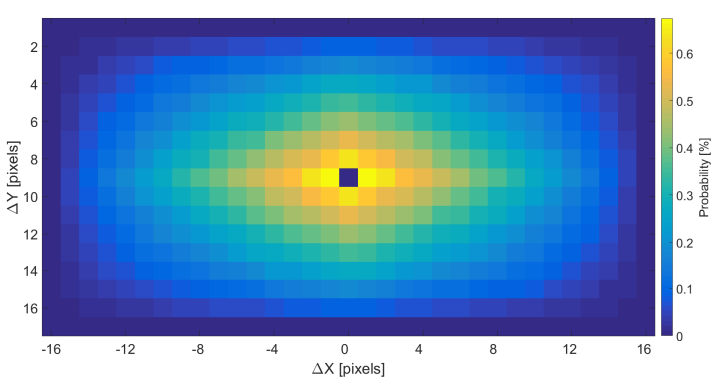

(a)

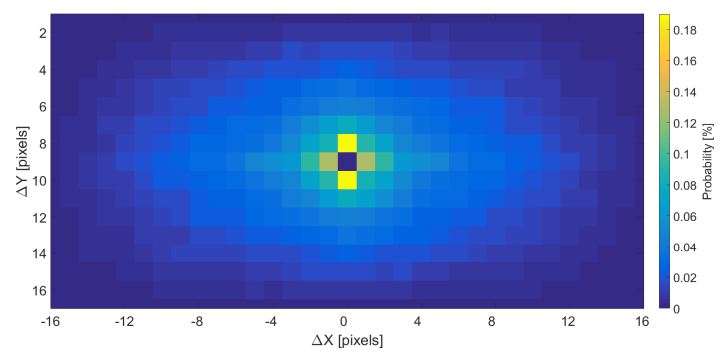

(c)

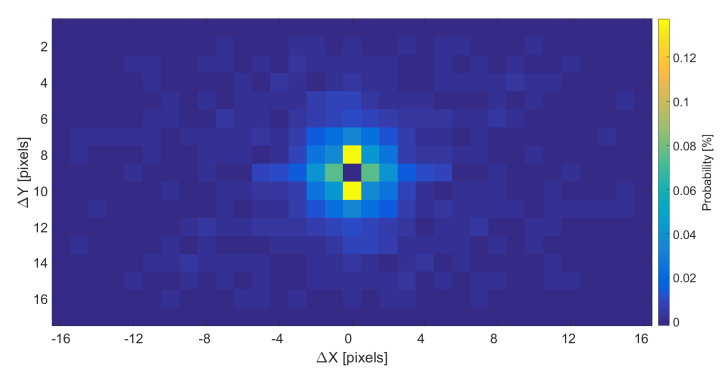

(e)

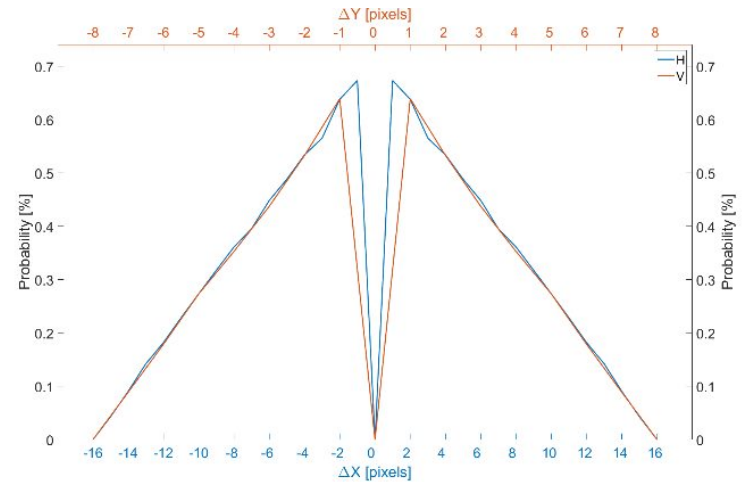

(b)

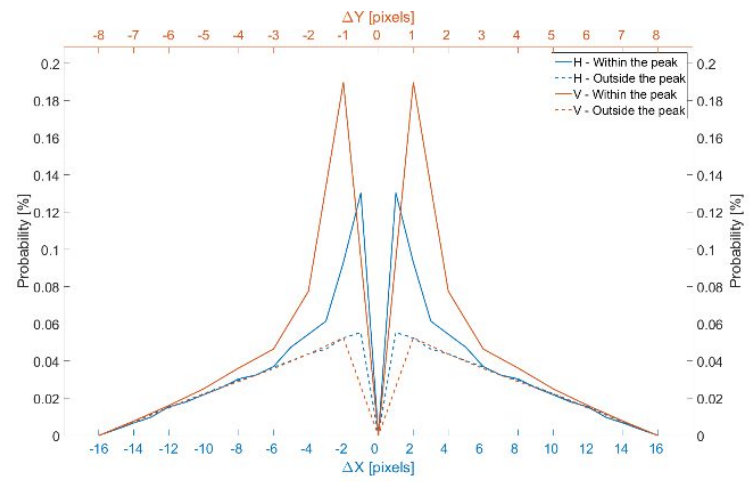

(d)

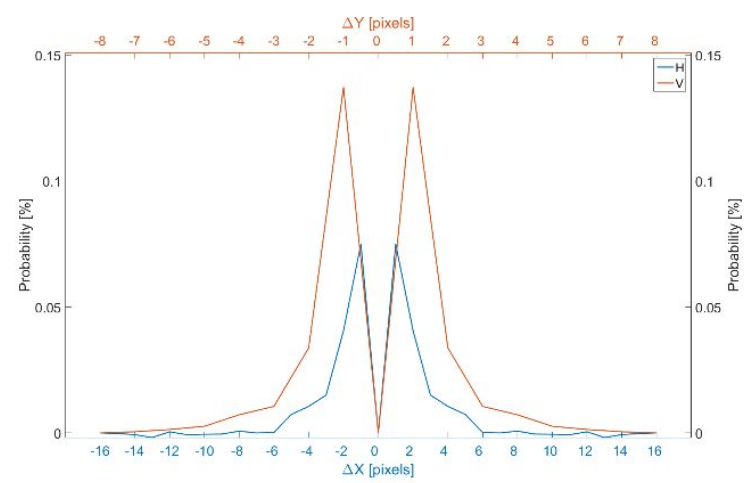

(f)

Figure 6 - Spatial correlations. (a) and (c) are obtained from the "tails" and "peak" datasets, respectively. (e) estimates the spatial correlations due crosstalk events only. It is obtained by estimating from the "tails" dataset the contribution of dark counts to the spatial correlations within the "peak" dataset and removing this contribution from (c). (b), (d) and (f) show the spatial correlation cross sections at $\Delta \mathrm{X}=0$ and $\Delta \mathrm{Y}=0$.

Spatial correlations have been calculated dividing the whole data into two sets, one corresponding to all couples with a difference in TDC code smaller than or equal to 0 (here referred to as the "peak" dataset), that includes dark counts and crosstalk events, the other with the remaining couples, containing dark counts only ("tails" dataset). From the "tails" dataset, a pyramidal distribution is obtained in Figure 6(a), as result of the 2D convolution of two uniform distributions. This is 
expected since dark counts are uniformly distributed across the pixels. The linear behavior is confirmed in Figure 6(b), showing the cross sections at $\Delta \mathrm{X}=0$ and $\Delta \mathrm{Y}=0$.

The "peak" data set exhibits a more-than-linear behavior in Figure 6(c). Such a crosstalk-induced distortion has a larger impact as the spatial difference approaches zero. Figure 6(d) shows the cross sections at $\Delta \mathrm{X}=0$ and $\Delta \mathrm{Y}=0$ along with the ideal linear behavior interpolated from the data in Figure 6(b) (dotted line), corresponding to a crosstalk-free sensor.

Figure 6(e) and Figure 6(f) show the spatial correlations due to crosstalk only, obtained by subtracting Figure 6(a) from Figure 6(c), after proper normalizations.

Crosstalk has a significant impact even at large distances. Residual spatial correlations are non-zero at up to $\Delta \mathrm{X}=4$ and $\Delta \mathrm{Y}=4$, i.e., at more than $1 \mathrm{~mm}$ distance. These values are surprisingly large if compared with other crosstalk studies [12]. This behavior can be ascribed to the presence of a glass carrier on top of the chip as typically required for Through Silicon Vias manufacturing and to the hot-carrier induced photon emission effect. Some of the emitted photons may exit the Silicon and reach distant pixels because of multiple reflections on the top glass.

\section{TOWARDS A NEW GENERATION OF SPAD-BASED IMAGERS FOR QUANTUM OPTICS APPLICATIONS}

SUPERTWIN aims at the development of a new all solid-state microscopy technique that exploits $\mathrm{N}$-partite photonic states to achieve super-resolution. SPAD-based imaging has been selected as the most promising technology to measure the spatial correlation patterns of the entangled photon backscattered from the target object.

A list of specifications for the imager include: (i) $\geq 256 \times 256$-pixel spatial resolution; (ii) temporal resolving capabilities with a resolution in the order of $100 \mathrm{ps}$; (iii) acquire data at a very high frame rate, synchronized with an external reference signal, e.g. a laser trigger running at $10 \mathrm{MHz}$; (iv) high sensitivity up to the near infra-red region of the spectrum, low dark count rates and negligible crosstalk; (v) ease of use and integration.

There is no market product, nor research work that fully satisfies these requirements. SUPERTWIN aims to develop a new imaging platform optimized for quantum physics experiments.

For state-of-the-art works, the size of each channel (called pixel) ranges from about $100 \mu \mathrm{m}$ down to $10 \mu \mathrm{m}$, with a decreasing trend as the technology evolves. The advantages of smaller pixels include (i) lower DCR, and (ii) better SPAD timing performance, since the capacitance reduces with the area. Pixels can be arranged in 2D arrays of sizes ranging from thousands up to almost 100,000 elements in most recent developments.

Efforts have been spent at manufacturing technology level to optimize the sensitivity and the noise performance. Recent results show a photon detection probability larger than $20 \%$ at $810 \mathrm{~nm}$, larger than $10 \%$ at $950 \mathrm{~nm}$, by means of a customized CMOS process [13]. This is typically achieved at the expense of poorer timing performance. With fully standard CMOS processes, the photon detection efficiency at those wavelengths is in the order of few percent, with a DCR density that can be as low as $0.17 \mathrm{~Hz} / \mu^{2}{ }^{2}$. Crosstalk is still a concern, as it creates spatially correlated artifacts in the measurement [12].

At the time of writing, the first SUPERTWIN SPAD imager design has been submitted for fabrication in fully standard 150nm CMOS process provided by LFoundry. The chip includes three different architectures that target the SUPERTWIN specifications. A first architecture is based on 
area-efficient TDCs, which represent a robust solution for recording the photon arrival times. A second architecture is based on the TDC analog counterpart, called Time-to-Analog Converter [14], which typically leads to more compact and energy efficient solutions at the expense of larger mismatches across the array. Both the two architectures implement a data compression scheme to reduce da data rate and increase the frame rate. A third, timestamp-less architecture aims to monitor the global photon activity and freeze the SPAD status if a number of photons have been detected simultaneously. The main advantage is the reduced amount of data being transmitted.

All the three architectures have $<50 \mu \mathrm{m}$ pixel pitch, thus enabling the implementation of $256 \times 256$ resolution imagers. According to the design, a $>20 \%$ pixel FF is obtained in all the architectures.

\section{ACKNOWLEDGEMENTS}

We thankfully acknowledge the support of the European Commission through the SUPERTWIN project, id. 686731.

\section{REFERENCES}

[1] Burri, S., Maruyama, Y., Michalet, X., Regazzoni, F., Bruschini, C., and Charbon, E., "Architecture and applications of a high resolution gated spad image sensor," Opt. Express 22, 17573-17589 (2014).

[2] Rojalin, T., Kurki, L., Laaksonen, T., Viitala, T., Kostamovaara, J., Gordon, K. C., Galvis, L., Wachsmann-Hogiu, S., Strachan, C. J., and Yliperttula, M., "Fluorescence-suppressed time-resolved raman spectroscopy of pharmaceuticals using complementary metal-oxide semiconductor (cmos) single-photon avalanche diode (spad) detector," Analytical and Bioanalytical Chemistry 408(3), 761-774 (2016).

[3] Braga, L. H. C., Gasparini, L., Grant, L., Henderson, R. K., Massari, N., Perenzoni, M., Stoppa, D., and Walker, R., "A fully digital $8 \times 16$ sipm array for pet applications with per-pixel tdes and real-time energy output," IEEE Journal of Solid-State Circuits 49, 301-314 (2014).

[4] Lussana, R., Villa, F., Mora, A. D., Contini, D., Tosi, A., and Zappa, F., "Enhanced single-photon time-of-flight 3d ranging," Opt. Express 23, 24962-24973 (2015).

[5] Jahromi, S., Jansson, J.-P., and Kostamovaara, J., "Solid-state 3d imaging using a 1nj/100ps laser diode transmitter and a single photon receiver matrix," Opt. Express 24, 21619-21632 (2016).

[6] Perenzoni, M., Perenzoni, D., and Stoppa, D., "6.5 a 64×64-pixel digital silicon photomultiplier direct tof sensor with $100 \mathrm{mphotons} / \mathrm{s} /$ pixel background rejection and imaging/altimeter mode with $0.14 \%$ precision up to $6 \mathrm{~km}$ for spacecraft navigation and landing," Proc. 2016 IEEE International Solid-State Circuits Conference (ISSCC), 118-119 (2016).

[7] Massari, N., Gasparini, L., Tomasi, A., Meneghetti, A., Xu, H., Perenzoni, D., Morgari, G., and Stoppa, D., “16.3 a $16 \times 16$ pixels spad-based $128-\mathrm{mb} / \mathrm{s}$ quantum random number generator with $-74 \mathrm{db}$ light rejection ratio and $-6.7 \mathrm{ppm} /{ }^{\circ} \mathrm{c}$ bias sensitivity on temperature," Proc. 2016 IEEE International Solid-State Circuits Conference (ISSCC), 292-293 (2016).

[8] STMicroelectronics, "Ds10285: Proximity and ambient light sensing (als) module." Datasheet http://www.st.com/resource/en/datasheet/vl6180x.pdf (2015).

[9] "Supertwin project website." http://www.supertwin.eu/.

[10] Dutton, N. A. W., Gyongy, I., Parmesan, L., Gnecchi, S., Calder, N., Rae, B. R., Pellegrini, S., Grant, L. A., and Henderson, R. K., "A spad-based qvga image sensor for single-photon counting and quanta imaging," IEEE Transactions on Electron Devices 63, 189-196 (2016).

[11] Krstajic, N., Levitt, J., Poland, S., Ameer-Beg, S., and Henderson, R., " $256 \times 2$ spad line sensor for time resolved fluorescence spectroscopy," Opt. Express 23, 5653-5669 (2015).

[12] Ficorella, A., Pancheri, L., Betta, G. F. D., Brogi, P., Collazuol, G., Marrocchesi, P. S., Morsani, F., Ratti, L., and Savoy-Navarro, A., "Crosstalk mapping in cmos spad arrays," Proc. 2016 46th European Solid-State Device Research Conference (ESSDERC), 101-104 (2016). 
[13] Takai, I., Matsubara, H., Soga, M., Ohta, M., Ogawa, M., and Yamashita, T., "Single-photon avalanche diode with enhanced nir-sensitivity for automotive lidar systems," Sensors 16(4), 459 (2016).

[14] Parmesan, L., Dutton, N. A., Calder, N. J., Krstajic, N., Holmes, A. J., Grant, L. A., and Henderson, R. K., “A $256 \times 256$ spad array with in-pixel time to amplitude conversion for fluorescence lifetime imaging microscopy," Proc. Int. Image Sensor Workshop, (2015). 